\title{
Self-Reported Non-Adherence and Beliefs About Medication in a Swedish Kidney Transplant Population
}

\author{
Annette Lennerling ${ }^{*}, 1,2$ and Anna Forsberg ${ }^{2,3}$ \\ ${ }^{1}$ Transplant Institute, Sahlgrenska University Hospital, Bruna Straket 5, $6^{\text {th }}$ floor, 41345 Gothenburg, Sweden \\ ${ }^{2}$ Institute of Health and Care Science, The Sahlgrenska Academy at University of Gothenburg, Box 457, 40530 \\ Gothenburg, Sweden \\ ${ }^{3}$ Department of Health Sciences at Lund University, Box 157, S-221 00 Lund, Sweden
}

\begin{abstract}
Background: Patients' non-adherence to immunosuppressant treatment after organ transplantation may lead to organ failure, graft loss and death. Non-adherence among Swedish kidney transplant recipients has not previously been studied. Hence the aim of this study was to explore non-adherence among Swedish kidney transplant recipients by using self-report instruments as well as testing the hypothesis that there is a difference in self-reported symptoms, beliefs about medicine and social support between respondents with or without self reported non-adherence.

Materials and Methodology: In the present cross sectional study 250 renal transplant recipients participated by replying to a questionnaire. Two validated instruments were included, one on beliefs about medicine (the BMQC), the other on nonadherence (the BAASIS $($ )).

Results: Only $46 \%$ never failed to follow the medical treatment with respect to taking the drugs, dosage or timing ( $>2$ hrs from prescribed time). Timing was the most frequently reported deviation (48\%). Forty-seven patients (16\%) had failed taking at least one dose of the prescribed immunosuppressants during the past four weeks. Four individuals had reduced the prescribed doses. Only one reported taking a 'drug holiday'. Nine participants reported stronger concerns than necessities for immunosuppressive medication. For the BMQ the necessity scores were extremely high while the scores for concern were low. Risk behaviour identified by the BAASIS had no association in risk attitudes as identified in the $\mathrm{BMQ}$. The only factor relating to non-adherence was lack of social support $(\mathrm{p}=0.022)$.
\end{abstract}

Conclusion: In general adherence was high. Identification of the exceptions remains a challenge.

Keywords: The BAASIS, the BMQ, immunosuppression, kidney transplant, life-long treatment, non-adherence, self-report instruments.

\section{INTRODUCTION}

Every day, transplant nurses establish numerous clinical encounters with organ transplant recipients (TxR), where one vital aspect is to empower the patient to cope with the lifelong immunosuppressive medication and its possible side effects. Since lack of empowerment and too many side effects might lead to non-adherence, the nurse's target is to view the patient as a person in a process of learning and to understand the perspective of the TxR in order to prevent non-adherence and its consequences. Until now, nonadherence in Swedish kidney transplant patients has not previously been studied. There was a need to establish a knowledge base about this complex phenomenon in relation to $\mathrm{TxR}$, in order to improve the everyday clinical meetings between the TxR and the transplant staff. Therefore, the starting point in this study was aimed at exploring nonadherence among Swedish kidney transplant recipients by the use of self-report instruments that also explored attitudes

*Address correspondence to this author at the Transplant Institute, Sahlgrenska University Hospital, Bruna Straket 5, 6th floor, 41345 Gothenburg, Sweden; Tel: +4631 342 7039;

E-mail: annette.lennerling@vgregion.se toward medication. We also wished to test the hypothesis that there is a difference in self-reported symptoms, beliefs about medication and social support between respondents with or without self reported non-adherence.

Patients' lack of adherence in various medical contexts has been carefully studied. However, a 1996 review concluded that there were few usable results for clinical use [1]. Adherence to long-term therapies has been defined as "the extent to which a person's behaviour - taking medication, following a diet, and/or executing lifestyle changes, corresponds with agreed recommendations from a health care provider" [2, p.17]. Despite this definition, health care professionals still act within a paradigm built on power, mastery and control [3] and we know from previous studies that only about half the patients with various chronic conditions requiring long-term therapies actually take their prescribed medication [2]. One such condition is being an organ transplant recipient, a state involving lifelong medication. From a nursing professional point of view, graft rejection is one of the most alarming consequences, which in worst case may lead to graft loss. It is well known that nonadherence to the immunosuppressive treatment after organ transplantation is associated with a poor outcome [4-6]. Dew et al., showed in a meta analysis of 147 studies that the rate 
of non-adherence in transplant recipients regarding immunosuppressive treatment, diet, exercise, and other healthcare requirements was 19 to 25 cases per 100 persons per year. Non-adherence to the immunosuppressive drugs was highest in kidney transplant recipients (36 cases per 100 persons per year) [7]. In a review article by Butler et al., on adherence to medication after renal transplantation, a median of $22 \%$ of the recipients (range, 18-26\%) were considered to be non-adherent. It was also stated that a median of $36 \%$ of the graft losses were associated with non-adherence (range, 14-65\%). There was a sevenfold increased risk of graft failure in the non-adherent patients compared with the adherent [4].

Several studies show that strict adherence to immunosuppressive drugs regarding taking, dosing and timing is required. For transplant recipients, even minor variations from the prescribed immunosuppressive therapy have been shown to be associated with an increased risk for poor outcome such as late acute rejections, poor function and graft loss [8-12]. In a prospective study with five years follow-up post kidney transplantation, $23 \%$ were identified as non-adherent, $21 \%$ of those experienced late acute rejection versus $8 \%$ of the adherent recipients [10].

A key problem is that it is difficult to discover nonadherence behaviour. Several methods such as blood-assays, pill count and prescription refill have been used, but no method is considered the gold standard clinically. Demographics, social support, and perceived health have proved little correlation with non-adherence [7], but it has been suggested that the attitude to medication and related beliefs are important factors for adherence to medical treatment [13]. We have therefore included that aspect in this study. The gold standard of measuring adherence is the use of electronic pill count, something that is unrealistic and unpractical to be used clinically. Even if the pills are counted there is no guarantee that they are actually taken. Various self-report instruments have been developed for the purpose of examining non-adherence and are described in a recent review article by Dobbels et al., [14]. It has been suggested that together with clinical judgement and blood-assays, selfreport instruments can be key components for assessment of adherence in clinical practice [15]. Thus the aim of this study was to explore non-adherence among Swedish kidney transplant recipients by using self-report instruments as well as testing the hypothesis that there is a difference in selfreported symptoms, beliefs about medicine and social support between respondents with or without self reported non-adherence.

\section{MATERIALS AND METHODOLOGY}

\section{Participants}

Sweden has over nine million inhabitants and Gothenburg is the second largest city in Sweden with the largest transplant centre in Scandinavia. Approximately 350 kidney transplantations are performed yearly in Sweden and close to half of them at Sahlgrenska University Hospital in Gothenburg. A registry of kidney transplant patients in Gothenburg was used to identify all recipients from January 2005 through June 2009 with functioning grafts and a follow-up time of at least six months. In March 2010, 348 persons were invited by mail to respond to a questionnaire.
A reminder was sent to those who had not responded after three weeks. In all, 258 questionnaires were received (74\% response rate), but as eight responders proved to have lost their grafts since last report, the study population comprised 250 persons. Demographic data and immunosuppressive therapy are presented in Table $\mathbf{1 .}$

Table 1. Demographic Data Regarding Gender, Age, First Transplant, Occupation, Level of Education and Immunosuppressive Therapy of the 250 Kidney Transplant Responders to the Questionnaire

\begin{tabular}{|c|c|}
\hline \multirow{2}{*}{\multicolumn{2}{|c|}{$\begin{array}{l}\text { Males, \% } 69 \\
\text { Age, years }\end{array}$}} \\
\hline & \\
\hline median, range & $54,19-77$ \\
\hline$\leq 30$ & 19 \\
\hline $30-59$ & 137 \\
\hline$\geq 60$ & 89 \\
\hline First kidney transplants, $\%$ & 86 \\
\hline \multicolumn{2}{|l|}{ Occupation, $\%$} \\
\hline employed, partly or full time & 44 \\
\hline sick-leave, full time & 27 \\
\hline retired & 15 \\
\hline unemployed & 8 \\
\hline students & 3 \\
\hline \multicolumn{2}{|l|}{ Education, \% } \\
\hline basic & 27 \\
\hline high school & 43 \\
\hline university & 29 \\
\hline \multicolumn{2}{|l|}{ Immunosuppressive therapy, $\%$} \\
\hline cyclosporine & 30 \\
\hline tacrolimus & 61 \\
\hline sirolimus or everolimus & 7 \\
\hline mycophenolate mofetil or & \\
\hline sodium mycophenolate & 88 \\
\hline prednisolone & 66 \\
\hline
\end{tabular}

\section{Data Collection}

Data were collected by a questionnaire that included two existing instruments - the Basel Assessment of Adherence with Immunosuppressive medication Scales (BAASIS@) [16] for which no validation data have been published and the Believes about Medicine Questionnaire (BMQ@) $[13,17,18]$. Dobbels et al., concluded in their review article that two self-report instruments could be recommended and we chose to use one of them in this study i.e. the BAASIS [14].

The BAASIS instrument was developed to assess adherence to immunosuppressive medication in adult transplant patients [16]. The instrument measures patients' taking, skipping, timing ( $>2 \mathrm{hrs}$ from prescribed time) and dose reduction of drugs. The recall period is limited to four weeks. The BAASIS comprises four questions with a 6-point scale for responses ranging from never (0) to every day (5). In addition, overall adherence is scored in percentage on a VAS scale ranging from 0 to 120 .

The BAASIS was developed for use in interviews but there is also a written questionnaire version [18]. In this study the latter was used. The BMQ was built on the 
theoretical framework that adherence decisions are influenced by a cost-benefit assessment. In this evaluation, personal beliefs about the necessity of the medication for maintaining or improving health are balanced against concerns about the potential adverse effects of taking it [13]. Agreement to use.

The items referring to taking medications in the BAASIS have been validated for adherence to anti-retrovirals in patients with HIV [19]. The validity of the items referring to taking and skipping medications for patients with HIV have been supported by Deschamps et al., [20]. Permission to use the BAASIS in this study was granted by Professor Sabina De Geest at the University of Basel in Basel, Switzerland.

The Beliefs about Medicine Questionnaire (BMQ) was published by Horne et al., in 1999 [13,17]. The BMQ was built on the theoretical framework that adherence decisions are influenced by a cost-benefit assessment. In this evaluation, personal beliefs about the necessity of the medication for maintaining or improving health are balanced against concerns about the potential adverse effects of taking it [13]. Agreement to use the BMQ has been obtained from Professor Rob Horne, University of London, UK (by Astellas Pharma Ltd Copenhagen). This instrument has been used and validated in the context of various chronic conditions requiring long-term medication. Respondents are requested to score $(1-5)$ the extent to which they disagree or agree to certain statements, from strongly disagree (1) to strongly agree (5). There are two parts in this instrument, one general and one specific. The general part contains 8 statements that can be divided into two subgroups regarding overuse, and harm of medication. The item correlation in the first subgroup, general harm, ranged from 0.81-0.47; and in the second subgroup, general overuse, it ranged from 0.71 0.51. In the specific part, 10 statements were divided into concerns and necessities regarding the drug treatment for the disease in question, in this case immunosuppressives. The item correlation in the subgroup concerns ranged from 0.80 0.39; and in the subgroup necessity it ranged from 0.81-0.65. The Cronbach's alpha values for the BMQ scales measured for various chronic diseases varied from $0.83-0.47$. The testretest reliability was considered to be within acceptable limits with correlations from $0.60-0.78$ [17]. No additional factor analysis was performed in this study.

For the purpose of the study, two author-constructed questions were added. The first question was How do the following sentences agree with your daily life as a kidney transplant recipient? This was followed by nine statements to which responses could range from I completely agree to I do not agree at all, all in analogue with the BMQ statements. The second question was How do the following sentences agree with your experienced situation? followed by 15 statements such as: "I have to cancel social activities often due to my condition," "My quality of life is very good," and "I often feel lonely." These statements covered the extent to which patients experienced trust in the health care system, insight in the treatment, quality of life, and social support. In addition, respondents were asked to select, from lists, their actual immunosuppressive medication and their perceived symptoms and side effects.

The statistical analyses were carried out in SPSS version 19. The Mann-Whitney rank test was used to evaluate differences between the two unpaired groups regarding age, number of side effects and scores for various beliefs and quality of life in respondents with or without self reported non-adherence.

The study was approved by the Regional Ethical Review Board in Gothenburg.

\section{RESULTS}

According to the BAASIS scoring system, scores of 1-5 on any of the four items, taking, skipping, timing and dose reduction, should be considered as non-adherence. With this definition, $46 \%(n=115)$ of the 250 patients were adherent. The most frequent aberration was timing, i.e. taking the drugs more than two hours away from the prescribed time. Time was cited by $48 \%(n=120)$. Of the 120 , one quarter 48 $\%(n=30)$ reported that this happened at least once a week. Of the 250 respondents, $36 \%(n=90)$ failed only in timing, while the others failed in more than one of the four aspects of non adherence.

More serious deviations to the prescribed regimen were acknowledged by $17 \%(n=44)$, all but one stating that they had omitted a dose of immunosuppressive medication during the past four weeks. Of those $44,86 \%(n=38)$ told that the omission had only occurred once, three had done it every other week and one once a week. "Drug holiday", i.e. skipping several doses in a row, was acknowledged by only one respondent. The last question in the BAASIS concerned taking less than the prescribed dose. Four individuals admitted having done so, two of them on a regular basis. According to the BAASIS instrument definition wherein deviation from even one of the items implies nonadherence, $54 \%(n=135)$ of the respondents were considered to be nonadherent.

In general, to be adherent to immunosuppressive drugs, more than $95 \%$ of the prescribed doses need to be taken to prevent graft rejection. The self-reported percentage of prescribed medication that was actually taken in the last four weeks was estimated on a scale ranging from $0-120 \%$, to cover overmedication. This estimation was built on the respondents' own perception of their medication taking. A total of 244 respondents answered this question. Among those, $75 \%(n=183)$ marked $100 \%$, suggesting complete adherence. Nine percent $(n=23)$ reported various values below $97 \%$.

The results of the BMQ questions are presented in Table 2. Among the scores for beliefs about medication in general, median values tended to be low for overuse and harm, whereas those for benefit were very high. For immunosuppressive medication specifically, the necessity score was extremely high, median 23 of 25 , while the concern scores were low. As suggested by the BMQ constructors, the difference between the necessity and concern scores was also calculated. The necessity-concerns differential may be thought of as the result of a cost-benefit analysis by each patient in which their perceptions of cost (concerns) are weighed against their perception of benefit (necessity beliefs). If the difference is positive, the patient perceives that the benefits of medication outweigh the costs. Four percent $(n=9)$ had values of zero or below, indicating stronger concern than sense of necessity. 
Table 2. Summary of Results for the General (G) and Specific (S), Sections of the Beliefs About Medicine Questionnaire

\begin{tabular}{|c|c|c|c|c|c|}
\hline Subgroups & (G) Overuse & (G) Harm & (G) Benefit & (S) Necessity & (S) Concern \\
\hline \hline Number of responses & 241 & 239 & 242 & 247 & 246 \\
\hline Max points & 20 & 20 & 20 & 23 & 11 \\
\hline Median & 10 & 9 & 17 & $9-25$ & $5-25$ \\
\hline Range & $4-17$ & $4-16$ & $5-20$ & & 25 \\
\hline
\end{tabular}

The frequency of various symptoms and side effects that might be related to the immunosuppressive therapy was high (Fig. 1). No symptoms or side effects were reported by only $11 \%(n=19)$ of the men and $13 \%(n=10)$ of the women. Fatigue was reported by $77 \%(n=192), 74 \%(n=185)$ had tremor, and $72 \%(n=180)$ weight gain. In general, women reported more symptoms than men, in particular insomnia, weight gain, changed bodily appearance, joint pain and hair loss; while acne and diarrhoea were the only symptoms reported more frequently by men. However the differences didn't reach statistical significance.

Age or gender did not differ between the adherent and non-adherent groups. Neither was there any difference between the groups with respect to the BMQ scores for overuse or concern or the necessity minus concern variable. Furthermore, the number of reported side effects was not increased in the non-adherent group. Out of six tested variables for quality of life, the extent of support from family and friends was more often classified as minor by the nonadherent patients $(\mathrm{p}=0.022)$.

\section{DISCUSSION}

Self reported adherence was surprisingly high in this population, as shown by the high scores on the beliefs of the necessities of the immunosuppressive treatment in the BMQ and also on the VAS scale in the BAASIS. Most kidney transplant persons taking part in this study appeared well informed about their medication and realised how important it was for their wellbeing. They were aware that they must take the drugs, although they knew that the drugs conferred side effects. Knowing this, we suggest that the transplant nurse approach the TxR in a non-paternalistic way and, instead of suggesting the risk for non-adherence, focus on developing educational strategies that TxR patients can use to grasp various aspects of the life long immunosuppressive therapy. Adopting such an inside perspective is in line with the recommendations of several researchers [21-24].

The two hour time difference from the prescribed regimen, as a single marker of non-adherence, seems to be too rigid and is not supported by sufficient scientific evidence. In a publication by De Geest et al., from 1998, timing was measured only with one drug, cyclosporine, and patients were already classified as major nonadherent. Further, the timing was measured with no upper time limit [8]. When using this criterion as a single marker, we found that too many patients are classified as non-adherent $(36 \%)$. Some deviation in time (i.e. $\pm 2 \mathrm{hrs}$ ) is common behaviour in their everyday life. The argument that such strict time limits

Men

Women

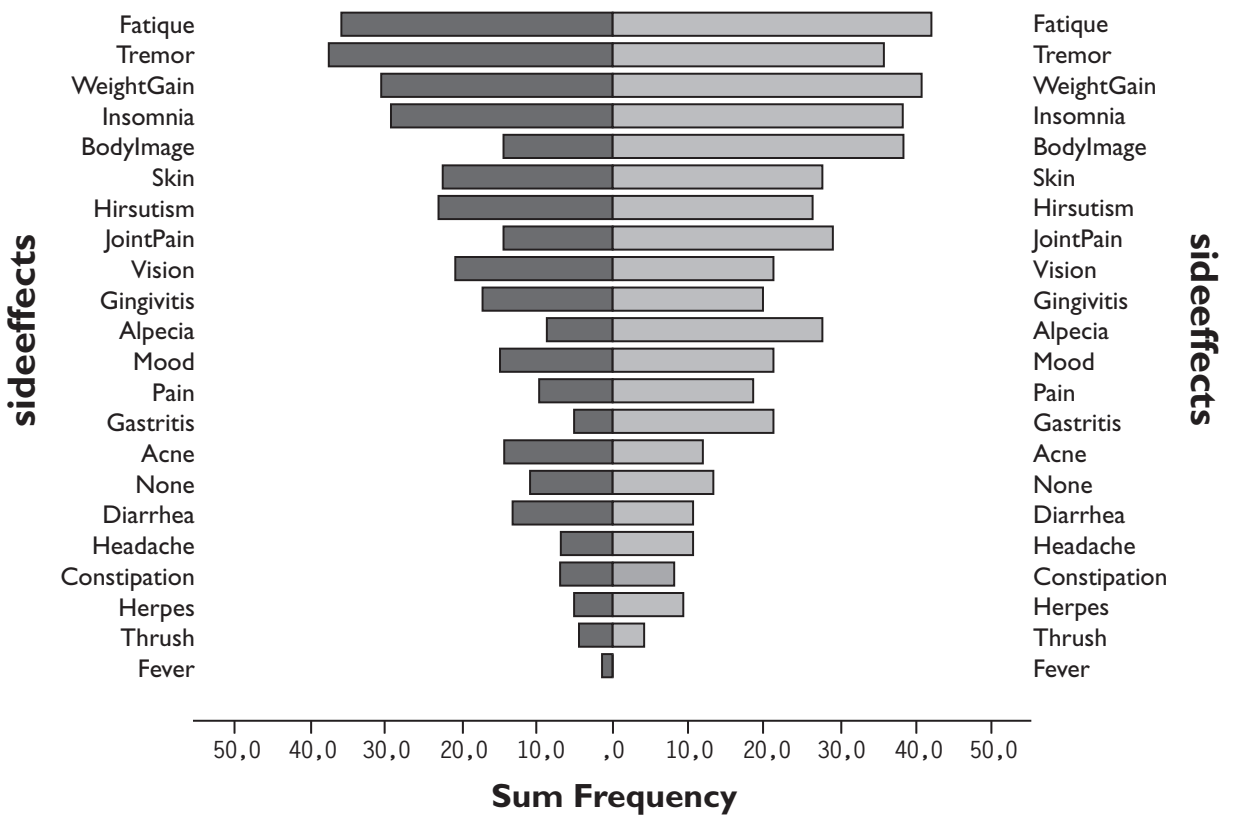

Fig. (1). The reported frequency of various symptoms and side effects that may be related to the immunosuppressive therapy divided by men and women $(\mathrm{n}=250)$. 
could compensate for underreporting is not clinically relevant due to the fact that most organ transplant recipients are prescribed double or triple therapy [16].

Based on the other variables from the BAASIS instrument, $17 \%$ of the patients in this study could be identified as non-adherent. No statistically significant relationships were found between BAASIS and the BMQ, gender and age. The only factor relating to self reported nonadherence was a sense of inadequate social support from family and friends. The same finding has been reported by Vlaminck et al., [10]. This suggests the need for nurses to assess the TxR's support system i.e. family and friends when followed up at the Out-patient Clinic and assess the risk for non-adherence on a regular basis and in each individual case.

In our population, it doesn't seem to be risk beliefs that cause non-adherence behaviour. This result differs from previous studies in patients with other chronic conditions e.g. asthma, renal failure or oncology patients [13]. In the study by Horn \& Weinman [13], medication beliefs were more powerful predictors of reported adherence than clinical and socio-demographic factors. The authors concluded that many patients engage in an implicit cost-benefit analysis in which beliefs about the necessity of their medication are weighed against concerns about the potential adverse effects of taking it and that these beliefs are related to medication adherence. The probable explanation for our results is that, in spite of their concerns, most patients realised the necessity of immunosuppressives for their wellbeing. They did not let their concerns influence their actual behaviour. A recent study by Nilsson et al., 2010 showed that there was a significant difference in the graft-related threat dimension $(\mathrm{p}<.000)$ between kidney transplant recipients $(\mathrm{KTx})$ and those who had received a liver, a heart or a lung [25]. Thus, the KTx's were more threatened by acute graft rejection than those transplanted with a liver, heart or lung. This difference remained significant even when the gender differences between the groups were controlled for. This could relate to the poor health related quality of life experienced when being on dialysis treatment [26], but it also suggests that their adherence to medication is good due to fear of losing the graft and being forced to be back on dialysis again. The transplant nurse needs to be sensitive to whether patients are acting out of fear instead of general contentment with their own capacity to master their health situations.

In the summary report from a 2008 consensus conference on non-adherence, the following definition of non-adherence was suggested "Deviation from the prescribed medication regimen sufficient to influence adversely the regimen's intended effect" [27]. This is a good definition, but the problem remains that there is no method of measuring how much immunosuppression is needed for the individual to prevent rejection. Some patients are certainly prescribed higher doses than they require, presumably causing various side-effects. Clinicians strive to develop methods for measuring and defining the target for immunosuppression on an individual basis. At the same time, nursing professionals need to develop a paradigm in which the patient is looked at as person within a learning process and attempt to understand the patient's behavior and actions in relation to medication. Conrad (1995) performed a today classic study about how patients with epilepsy perceive medication and argued that the result might be generalised to apply to many other patients with chronic conditions demanding regular medication [21]. The results showed that persons with an illness or disease are self-regulating rather than adherent and that a strategy that, from a medical perspective, might be viewed on as non-adherent in fact is a way of achieving a sense of control over the disease. This is in line with the results of a study of perceptions of experiences of graft rejection among organ transplant recipients showing that they clearly strived to control the uncontrollable [28]. We suggest a 3-step person centered approach: listening to the patient's illness narrative, establishing patient participation in decision making regarding their treatment and care plan, and documenting the patient's preferences and needs in the medical record $[29,30]$. This approach makes it more likely that barriers to adherence will be revealed. After mutual discussions between the patient and the care giver, possibilities to overcome the identified barrier can be suggested.

\section{STUDY LIMITATIONS}

There are several limitations in this study. One might argue that the study lacks originality, as there are similar research papers with the same or better design. However, a similar research was never done in Sweden and from a nursing perspective this is a highly relevant matter. The design is cross sectional, which limits interpretation of the results. A bias may also be that the non-respondents were more nonadherent. The self-report questionnaire was the only measurement used, but since self-report instruments can be a key component for assessment of adherence in clinical practice we believe the instrument was appropriate. In retrospect, a factor analysis would have been useful. Another weakness concerns BAASIS wherein a recall period of 4 weeks might be too long, leading to less response accuracy. We also need to consider the low but possible temptation among the respondents to try to meet the researcher's expectations by being a good patient and give the "expected" answer.

The study population was Swedish, and factors such as the health care system and national level of education certainly influence adherence. Finally, from a medical perspective, the gold standard in adherence research at the present time is an electronic pill count. Other methods are judged as inferior. Unfortunately this gold standard also reflects a paradigm of power, mastery and control that we wish to avoid; therefore the pill count was never considered as a methodological option.

\section{CONCLUSION}

In conclusion, self-reported non-adherence among Swedish kidney transplant recipients was low when not involving the 2-hour time difference from the prescribed regimen. In spite of their concerns, most patients realised the necessity of immunosuppressives for their post-transplant wellbeing. Despite the overall high frequency of various symptoms and side effects that might be related to the immunosuppressive therapy, the number of reported side effects was not increased in the non-adherent group. Instead, the extent of support from family and friends was significantly more often classified as minor by the nonadherent patients, indicating that social support might be a 
predictor for non-adherence among kidney transplant recipients while symptom burden is not. Further research will involve using grounded theory to explore the main concerns in relation to social support and adherence among organ transplant recipients.

\section{ACKNOWLEDGMENT}

Swedish Kidney Patient Association (Njurförbundet) for cooperation.

Lena Heidenberg for initial administrative help (A. Lennerling).

Professor Gudrun Nyberg for valuable comments and input during the analyses and writing process (A. Lennerling).

Astellas Pharma Ltd Copenhagen for financial support performing the study (for fees for the application to the Ethical Review Board, for the use of the questionnaire BMQ $\odot$ and for mailing costs. (A. Lennerling).

Lennart Jacobsson testamente for grant preparing the manuscript (A. Lennerling).

Professor Michael Olausson for financial support preparing the manuscript (A. Lennerling).

The study was performed within the Forsberg \& Lennerling Research group in organ donation and organ transplantation.

\section{CONFLICT OF INTEREST}

Declared none.

\section{REFERENCES}

[1] McGavock H. A review of the literature on drug adherence. Royal Pharmaceutical Society of Great Britain. Hoddesdon. UK: Merck Sharp and Dohme Ltd 1996.

[2] Adherence to long-term therapies. Evidence for action. Geneva: World Health Organization WHO 2003. Available at: http://apps.who.int/ medicinedocs/en/d/Js4883e/

[3] Buchanan D. An Ethic for Health Promotion: Rethinking the Sources of Human Well-being. New-York: Oxford University Press 2000.

[4] Butler JA, Roderick P, Mullee M, Mason JC, Peveler RC. Frequency and impact of nonadherence to immunosuppressants after renal transplantation: a systematic review. Transplantation 2004; 77: 769-76.

[5] Denhaerynck K, Dobbels F, Cleemput I et al. Prevalence, consequence, and determinants of nonadherence in adult renal transplant patients: a literature review. Transplant Int 2005; 18: 1121-33.

[6] Morrissey PE, Flynn ML, Lin S. Medication noncompliance and its implications in transplant recipients. Drugs 2007; 67: 1463-81.

[7] Dew MA, DiMartini AF, De Vito Dabbs A et al. Rates and risk factors nonadherence to the medical regimen after adult solid organ transplantation. Transplantation 2007; 83: 858-73.

[8] De Geest S, Abraham I, Moons P et al. Late acute rejections and subclinical noncompliance with cyclosporin therapy in heart transplant recipients. J Heart Lung Transplant 1998; 17: 854-63.

[9] Nevin TE, Kruse L, Skeans MA, Thomas W. The natural history of azathioprine compliance after renal transplantation. Kidney Int 2001; 60: $1565-70$.
[10] Vlaminck H, Maes B, Evers G et al. Prospective study on late consequences of subclinical non-compliance with immunosuppressive therapy in renal transplant patients. Am J Transplant 2004; 4: 1509-13.

[11] Takemoto SK, Pinsky BW, Schnitzler MA et al. A retrospective analysis of immunosuppression compliance, dose reduction and discontinuation in kidney transplant recipients. Am J Transplant 2007; 7:2704-11.

[12] Nevin TE, Thomas W. Quantitative patterns of Azathioprine adherence after renal transplantation. Transplantation 2009; 87: 711-8.

[13] Horne R, Weinman J. Patients' beliefs about prescribed medicines and their role in adherence to treatment in chronic physical illness. J Psychosom Res 1999; 47: 555-67.

[14] Dobbels F, Berben L, De Geest S et al. The Transplant360 Task Force. The psychometric properties and practicability of self- report instruments to identify medication nonadhernece in adult transplant patients: A systematic review. Transplantation 2010; 90: 205-19.

[15] Schäfer-Keller P, Steiger J, Bock A, Denhaerynck K, De Geest S. Diagnostic accuracy of measurement methods to assess non-adherence to immunosuppressive drugs in kidney transplant recipients. Am J Transplant 2008; 8: 616-26.

[16] BAASIS $\odot$ Leuven-Basel research group Institute of Nursing Science University of Basel 2005. Personal communication with Professor Sabina De Geest September $15^{\text {th }} 2009$.

[17] Horne R, Weinman J, Hankins M. The beliefs about medicines questionnaire: the development of a new method for assessing the cognitive representation of medication. Psychol Health 1999; 14: 1-24.

[18] Horne R, Frost S, Hankins M, Wright S. In the eye of the beholder: pharmacy students have more positive perceptions of medicines than students of other disciplines. Int J Pharm Pract 2001; 9: 85-89.

[19] Glass TR, De Geest S, Weber R et al. and the Swiss HIV cohort Study. Correlates of adherence in HIV-infected patients receiving antiretroviral therapy: the Swiss HIV Cohort Study. J Acquir Immune Defic Syndr 2006; 41: 385-92.

[20] Deschamps A, De Geest S, Vandamme AM, Bobbaers H, Peetermans WE, Van Wijngaerden E. Diagnostic value of different adherence measures using electronic monitoring and virological failure as reference standards. AIDS Patient Care STDS 2008; 22: 735-43.

[21] Conrad P. The meaning of medications: another look at compliance. Soc Sci Med 1985; 20: 29-37.

[22] Donovan JL, Blake DR. Patient non-compliance: deviance or reasoned decision-making? Soc Sci Med 1992; 34: 507-13.

[23] Friberg F, Pilhammar AE, Bengtsson J. Pedagogical encounters between nurses and patients in a medical ward: a field study. Int J Nurs Stud 2007; 44: 534-44.

[24] Friberg F, Öhlén J. Searching for knowledge and understanding while living with impending death - a phenomenological case study. Int J Qual Health Well-being 2007; 2: 217-26.

[25] Nilsson M, Forsberg A, Bäckman L, Lennerling A, Persson L-O. The perceived threat of the risk for graft rejection and health related quality of life among organ transplant recipent's. J Clin Nurs 2011; 20: 274-82.

[26] Terada I, Hyde C. The SF-36: an instrument for measuring quality of life in ESRD patients. EDTNA ERCA J 2002: 28:73-6, 83.

[27] Fine RN, Becker Y, De Geest $\mathrm{S}$ et al. Nonadherence consensus conferens summery report. Am J Transpl 2009; 9: 35-41.

[28] Nilsson M, Persson L-O, Forsberg A. Perceptions of experiences of graft rejection among organ transplant recipients - striving to control the uncontrollable. J Clin Nurs 2008; 17: 2408-17.

[29] Ekman I, Swedberg K, Taft C et al., Person-centered care--ready for prime time. Eur J Cardiovasc Nurs 2011; 4: 248-51.

[30] Sandman L, Granger BB, Ekman I, Munthe C. Adherence, shared decision-making and patient autonomy. Med Health Care Philos 2011; Jun 17 [E-pub ahead of print]. 